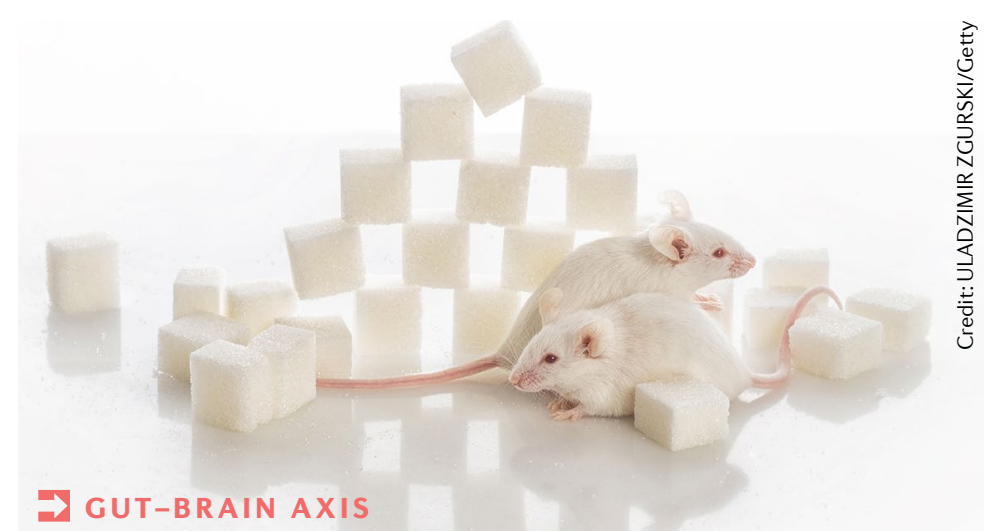

\title{
A gut instinct for glucose
}

All animals use sugar to produce energy, and so it is not surprising that they have evolved a preference for sugar over non-nutritional sweeteners. However, even animals that lack the sugar-sensitive taste receptors T1R2 and T1R3 develop a preference for sugar over sweeteners, suggesting that sugar preference can be mediated via means besides taste. Now, writing in Nature, Tan et al. characterize a gut-brain connection that is needed for sugar preference in mice.

The authors compared labelling of FOS protein (a marker of neuronal activity) in the brains of mice after drinking different solutions. They identified a population of neurons in the caudal nucleus of the solitary tract (cNST) that was activated after consuming sugar solution but was not activated after consuming water or a sweetener solution. Calcium imaging of cNST neurons during intragastric infusion of the solutions confirmed that these neurons respond to the infusion of glucose solution but not to the infusion of water or sweetener solution. The glucose responses in these cNST neurons were prevented by transection of the vagus nerve, suggesting that the presence of sugar in the gut is signalled by the vagus nerve to the cNST.

Next, the authors used TRAP (targeted recombination in active populations) to selectively express Cre recombinase in sugar-activated cNST neurons, and injected a Credependent monosynaptic retrograde reporter into the cNST, to map the inputs to these cells. This approach revealed that the sugar-activated cNST neurons receive direct inputs from neurons in the vagal ganglion.
Silencing sensory neurons in the vagal ganglion, through injection of a virus encoding tetanus toxin, prevented mice from developing a preference for drinking sugar solution over sweetener solution. Moreover, mice in which sugarresponsive cNST neurons were selectively silenced (using TRAP and a Cre-dependent virus encoding tetanus toxin) no longer had a preference for sugar over sweetener but still retained their preference for sweet-tasting solutions over water. Therefore, this gut-cNST connection is required for the preference for sugar.

The authors imaged calcium signals in vagal sensory neurons in the vagal ganglion while the intestine was infused with different solutions. Whereas selective vagal ganglion neurons were activated less than 30 seconds after intestinal infusion with solutions containing either glucose or a glucose analogue, very few of these neurons were activated following infusion with sweetener solution. Moreover, $20 \%$ of the vagal afferent neurons labelled using a fluorescent cholera toxin tracer injected into the duodenum were responsive to glucose infusion.

Previous research showed that mice lacking T1R2 and T1R3 still develop a preference for sugar over sweetener. The authors therefore reasoned that there must be another receptor for sugar in the gut. Sodium-glucose-linked transporter 1 (SGLT1) is the main glucose transporter in the gut, making it a possible candidate. Indeed, infusion of the other main substrates of SGLT1 besides sugar
- namely, galactose and the glucose analogue 3-OMG - activated sugar-responsive vagal neurons, and blocking intestinal SGLT1 with its antagonist phlorizin abolished vagal neuron responses to infusion of glucose. Thus, SGLT1 is necessary for transducing sugar signals in intestinal vagal neurons.

To be able to genetically target the sugar-responsive cNST neurons, the authors consulted the Allen Brain Atlas for candidate genes that might define this population of neurons. More than $80 \%$ of the neurons in the cNST that express proenkephalin (Penk) showed responses to intestinal sugar infusion, and conversely, about $85 \%$ of cNST neurons activated following sugar infusion expressed Penk, suggesting that Penk expression is a good marker for these neurons.

Finding this marker enabled the authors to chemogenetically target these sugar-responsive cNST neurons. The authors gave mice expressing the excitatory designer receptor hM3Dq in Penk ${ }^{+}$cNST neurons two differently flavoured bottles of water, with one flavour sweeter than the other. As expected, the mice innately preferred the bottle with the sweeter flavour. The authors then included clozapine - the agonist of hM3Dq - in the bottle containing the less preferred flavour, to activate the sugar-preference circuit. This manipulation led to the animals forming a preference for the initially less preferred flavour. Therefore, the activity of the sugar-responsive cNST neurons mediates the formation of sugar preference, and can be 'hijacked' to create novel preferences.

Together, these results demonstrate that a group of SGLT1expressing cells in the gut signal the presence of sugar in the gut to a population of cNST neurons, which in turn can mediate the formation of a preference for ingested sugar. As this pathway is not activated by artificial sweeteners, it might help to explain why the introduction of such sweeteners in sweet foods has done little to reduce preference, and appetite, for sugar.

Natasha Bray nerve to the cNST

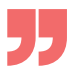

ORIGINAL ARTICLE Tan, H.-E. et al. The gutbrain axis mediates sugar preference. Nature $\mathbf{5 8 0}$, 511-516 (2020) 